\title{
LAS DINÁMICAS DE GÉNERO EN LAS BRIGADAS FEMENINAS DE LA GUERRA SIRIA
}

\section{GENDER DYNAMICS IN THE WOMEN'S BRIGADES OF THE SYRIAN WAR}

\author{
Isabel García \\ Universidad Nacional de Educación a Distancia
}

\section{RESUMEN}

Tras el estallido de la guerra siria en el año 2011 varios grupos que se dan cita en el contencioso crearon sus propias brigadas femeninas, consiguiendo una rápida proyección internacional y un interés inusitado por los medios de comunicación de masas. Este artículo contribuye a explicar este repentino aumento de mujeres combatientes dentro del contexto del país árabe. Para ello se llevará a cabo una comparación entre los cuatro principales grupos que se dan cita en la guerra: Daesh, el Ejército Libre Sirio, las Unidades de Protección Popular y las fuerzas gubernamentales. El artículo aborda la ideología de género de estas organizaciones y explica las posibles razones que les han llevado a contar con presencia femenina en sus filas.

Palabras clave: mujer, género, Siria, guerra, terrorismo.

\begin{abstract}
After the outbreak of the Syrian war in 2011 , several groups created their own women's brigades. As a result, there was a rapid rise in the international coverage and an unexpected interest from the mass media. The aim of this article is to shed light on this phenomenon within the context of the Arab country. To that end this, the research will make a comparison between the four main groups in the war: Daesh, the Free Syrian Army, the Syrian Kurdish Force and the Assad regime. Finally, the article deals with the gender ideology of these organizations and explains the possible reasons which have led them to have a female presence in their ranks.
\end{abstract}

Keywords: women, gender, war, Syria, terrorism. 


\section{RESUM \\ LES DINÀMIQUES DE GÈNERE A LES BRIGADES FEMENINES DE LA GUERRA A SIRIA}

Després de l'esclat de la guerra siriana l'any 2011 diversos grups que es donen cita en el contenciós crearen les seues pròpies brigades femenines, aconseguint una rápida projecció internacional i un interès inusitat pels mitjans de comunicació de masses. Aquest article contribueix a explicar aquest augment sobtat de dones combatents dins del context del país àrab. Amb la qual cosa, es durà a terme una comparació entre els quatre principals grups que es donen cita a la guerra: Daesh, I'Exèrcit Lliure Sirià, les Unitats de Protecció Popular i les forces governamentals. L'article aborda la ideología de gènere d'aquestes organitzacions i explica les possibles raons que els han portat a comptar amb presència femenina a les seves files.

Paraules clau: gènere, Siria, guerra, terrorisme. 
Las mujeres han participado a lo largo de la historia en diferentes conflictos armados y pese a que, generalmente, su actuación ha estado relegada a la retaguardia, existen ejemplos de mujeres que tuvieron una parte más activa en el combate. Algunas de estas pioneras figuras de la época contemporánea fueron las francotiradoras soviéticas o las mujeres británicas que sirvieron como pilotos durante la II Guerra Mundial (VINOGRADOVA, 2017; YEGINSU, 2018). Tampoco se quedaron atrás las mujeres que participaron en las diferentes luchas anticoloniales, siendo el caso de Argelia uno de los más reconocidos (DJAMILA y ABU-HAIDAR, 1999: 62). Sin embargo, el papel de estas ha ido evolucionando en función de la cultura propia del entorno donde se disputaba la acción y conforme las contiendas tradicionales iban dando paso a los conocidos como conflictos asimétricos. El rol que ha desempeñado la mujer en estas ofensivas se ha analizado desde diferentes perspectivas sin embargo, su contribución a la lucha armada sigue siendo desconocida pese a que su presencia es cada vez más relevante, sobre todo dentro de grupos insurgentes o de carácter terrorista. Así, El Ejército Rojo Japonés, ETA, Sendero Luminoso o Los Tigres de Liberación de Eemal Tamil (LTTE, por si siglas en inglés) son solamente algunos 
ejemplos de las organizaciones que han contado con mujeres entre sus miembros. No obstante, la gran mayoría de las investigaciones sobre el fenómeno han centrado su atención en los hombres, asumiendo que el papel que desempeñaban las mujeres era secundario (CUNNINGHAM, 2003).

Las primeras investigaciones en los años 70 y 80 pusieron su foco de atención en la participación femenina en la revolución rusa y en las guerrillas de América Latina. Una década después la comunidad científica amplió su atención de forma visible hacia las mujeres que eran miembros del IRA. Durante los años 90 solamente se publicaron dos libros que se adentraban en explicar la participación de mujeres en movimientos extremistas y las razones psicológicas y sociológicas que había detrás de su adhesión a los grupos (MCDONALD, 1991; DE CATALDO y TITIANA, 1992).

Es a partir del año 2000 cuando aumenta el número de publicaciones, fecha que coincide con la perpetración de actos terroristas suicidas por parte de mujeres de forma asidua. Se trata de uno de los temas que más ha preocupado a la comunidad internacional y hay varios autores que han tratado de estudiar esta emergencia en conflictos como Chechenia, Palestina o Irak (SPECKHARD, 2008; O'ROURKE, 2009). No obstante, hay una discrepancia patente sobre cuáles son las principales motivaciones que conducen a las mujeres a verse envueltas en actos violentos. Una de estas corrientes sostiene que la principal razón atiende a la vertiente personal y que los vínculos familiares, la pérdida de un ser querido o haber sufrido una agresión sexual son los motivos más recurrentes (VICTOR, 2004).

Esta relevancia dentro del campo académico por la mujer y la violencia volvió a despuntar tras el estallido del conflicto sirio gracias a la creación de diferentes brigadas femeninas. En 2013 la cifra de mujeres que se había unido a grupos rebeldes en la región se estimaba en 5.000 (SHOLMAN, 2013). La investigadora Sjoberg sostiene que la participación de las mujeres en el contencioso ha conseguido más cobertura mediática que la mayoría de las guerras civiles pero que aún se desconocen las motivaciones que han tenido para vincularse a los diferentes grupos dentro del conflicto (SJOBERG, 2018).

Este artículo contribuye a explicar este repentino aumento de mujeres combatientes dentro del contexto del país árabe. Para ello se estudian las razones que propiciaron el cambio a través de los cuatro principales grupos que se dan cita en el contencioso. Estas organizaciones son: Daesh, Unidades de Protección Popular (YPG, en sus siglas en kurdo), El Ejército Libre Sirio (ELS) y las fuerzas gubernamentales de Bashar al-Asad. Se han elegido estos grupos debido al interés mediático que han suscitado las mujeres de estas organizaciones, en especial las militantes de Daesh (SJOBERG, 
2018: 301). El artículo aborda, siempre que se cuente con información disponible, el papel beligerante de la mujer dentro de estos grupos y la ideología de género que estas organizaciones han trasladado a la opinión pública.

Resulta imprescindible señalar que la investigación se enfrenta con una importante dificultad a la hora de recopilar información ya que hay pocos estudios al respecto debido al reciente suceso de los acontecimientos. Se ha tratado de solventar esta problemática analizando la propaganda vertida por cada uno de los actores analizados, como las revistas oficiales de Daesh (Dabiq o Rumiyah). También han servido de base documental diferentes fuentes secundarias, bases de datos e informes de reputadas organizaciones internacionales como Freedom House, Naciones Unidas o Human Rights Watch. El artículo comienza con el estallido de la revolución y una aproximación a la situación de la mujer en la región antes y durante el inicio de las protestas.

\section{EL ESTALLIDO DE LA REVOLUCIÓN Y LA CONQUISTA DE NUEVOS ESPACIOS FEMENINOS}

El conflicto sirio estalló en el año 2011 al amparo de las manifestaciones populares conocidas como Primaveras Árabes. Pese al carácter pacífico y democrático que tuvieron las primeras protestas en el país, lo cierto es que poco a poco fueron derivando en una confrontación civil a gran escala. La población ha sido masacrada durante ocho años y se ha cobrado la vida de alrededor de 511.000 personas. Además, la violencia y el sectarismo característicos del conflicto han provocado el desplazamiento de casi siete millones de personas dentro de Siria y de más de cinco fuera de sus fronteras, desencadenando en una de las mayores crisis humanitarias que se recuerdan en pleno siglo XXI (HRW, 2018).

En este contexto de caos se han dado cita una gran diversidad de actores que no han hecho más que recrudecer la fragmentación social que impera en el país. Así, coexisten diferentes milicias armadas como el Ejército Libre Sirio (ELS), facciones como los peshmerga kurdos o las Unidades de Protección Popular (YPG). Estas, a su vez, disputan protagonismo con los dos principales grupos yihadistas que operan en la zona: Daesh y Jabhat al-Nusra, filial de Al Qaeda en Siria y recientemente bautizado con el nombre de Hayat Tahrir al-Sham (ÁLVAREZ-OSSORIO, 2016). Mientras, las fuerzas gubernamentales de Bashar al-Asad intentan recuperar el control de un territorio roto.

La reciente creación de brigadas femeninas por parte de estos grupos ha permitido a las mujeres escalar posiciones dentro de la esfera pública siria. Hasta ese momento dominada por los hombres, debido, en parte, a que la 
sociedad siria se rige por unas normas culturales bastante conservadoras (CHARLES y DENMAN, 2012: 205). Así, aunque la constitución garantiza la igualdad de género, lo cierto es que algunas leyes siguen perpetuando la discriminación femenina en la República árabe. Por ejemplo, aún en el año 2011 el régimen permitía penas indulgentes para los delitos cometidos en nombre del conocido como 'crimen de honor'. Además, en el país no se contempla el delito de violación dentro del matrimonio e incluso existe la posibilidad de que los violadores puedan casarse con su víctima para conmutar su pena (UNICEF, 2011).

No existe una brecha de género con respecto a la educación. El 92\% de las mujeres y el $97 \%$ de los hombres poseen estudios de educación primaria en el país (CHARLES y DENMAN, 2012: 204). Estas, además, representan el $46 \%$ del alumnado universitario aunque sus preferencias a la hora de elegir carrera siguen centrándose en campos asociados a la Educación y la Literatura. Los estudios vinculados con ingenierías o Medicina cuentan con menor presencia femenina (Freedom House, 2010). Estos datos de representación no se trasladan al mercado laboral (NASSER y MEHCHY, 2012: 2). Y es que un año antes de que estallaran las protestas, solamente el 12 , $9 \%$ de mujeres mayores de 15 años eran población activa. Los autores de dicho estudio sostenían que el matrimonio era un factor determinante para explicar dicha exclusión. ' No se trata de ninguna coincidencia, por tanto, que el país ostentara el puesto 124 de 135 países en el Índice Global de la Brecha de Género del año 2011 (HAUSMANN, TYSON y ZAHIDI, 2011). El estallido de la revolución rompió con tabúes arraigados en la sociedad siria y durante los primeros meses del levantamiento las mujeres participaron activamente en las protestas. ${ }^{2}$ Estas formaron parte de las demostraciones públicas, se organizaron alrededor de diferentes grupos como Syrian Women for the Syrian Intifada, establecieron redes para abastecer de ayuda humanitaria a la población civil (GHAZZAWI, 2014) e incluso denunciaron públicamente las atrocidades del régimen. Este fue el caso de la activista Razan Zaitouneh (CHARLES y DENMAN, 2012: 197), que se

1 Siria es uno de los países árabes que registra mayor tasa de matrimonios infantiles. Para más información consultar "A profile of child marriage in the Middle East and North Africa" (2016), Unicef.

2 Las mujeres sirias han estado involucradas en el activismo durante años y las organizaciones feministas tuvieron un considerable crecimiento durante la presidencia de Bashar al-Asad. Para más información sobre el tema consultar a Jeanne, 2015. 
vio obligada a vivir en la clandestinidad por temor a las represalias del régimen. Asimismo, fue una mujer, la abogada Muntha al-Atrash, la primera que se atrevió a pedir públicamente la dimisión del presidente sirio en abril de 2011 (JEANNE, 2017: 236). Este gesto sirvió de inspiración para muchas mujeres que con el incremento de la violencia y de la represión ejercida en numerosas ocasiones por parte del Estado, fueron adaptándose a las nuevas circunstancias del conflicto, asumiendo en algunos casos el rol de combatientes.

\section{LAS MILITANTES DE DAESH: LA NUEVA ERA DEL YIHADISMO FEMENINO}

Daesh es un grupo terrorista de carácter transnacional que propone, con unas técnicas depuradas y modernas, un modelo de Estado en el que son necesarios hombres y mujeres para su puesta en marcha. Su origen fundacional es Al Qaeda en Irak (AQI). Este grupo insurgente iraquí, que se encontraba en declive en el año 2011, ve una oportunidad de expansión en territorio sirio tras el estallido de la Primavera Árabe en la nación vecina. El grupo supo aprovechar el caos en el que estaba sumido Siria, así como el descontento de gran parte de la población de la zona rural con las políticas de Bashar al-Asad para ganar apoyos y apoderarse de un amplio territorio.

La organización consiguió una conquista relativamente rápida y en junio de 2014 Abu Bakr al-Baghdadi se autoproclamó líder de todos los musulmanes y anunció el establecimiento de un califato cuya capital se asentaría en la ciudad siria de Raqa. Desde entonces y hasta el año 2016, fecha en la que comienza su declive territorial, la organización llegó a controlar varias provincias de Siria e Irak y ciudades tan importantes como Mosul. El grupo gestionó esta región, compuesta por seis millones de habitantes, con un sistema administrativo bastante eficiente, fundamentado en su estricta interpretación de la sharía (AVILÉS, 2017: 176). Así, la organización impuso normas sociales de segregación por sexo, estableció estrictos códigos de vestimenta para las mujeres y prohibió escuchar música occidental o consumir alcohol. Bajo el califato también se instauró un mercado de esclavas sexuales cuyas víctimas fueron principalmente mujeres y niñas pertenecientes a la minoría yazidí. ${ }^{3}$

3 Los yazidíes pertenecen a una minoría religiosa preislámica. Hasta el siglo VII d.C fue la religión oficial de los kurdos. 
Daesh ha regulado su política de género a través de su aparato de propaganda y su visión con respecto a la violencia ejercida por las mujeres también ha quedado reflejada en su estrategia de comunicación. El artículo $A$ jihad withouth fighting (AL-MUHAJIRAH, 2015: 40-45) apunta de manera explícita que su ideal es que las mujeres deben estar alejadas del campo de batalla. No obstante, el grupo sostiene también a través de varios documentos que la violencia es una opción para las mujeres en caso de defensa propia o si no hay hombres suficientes para proteger el territorio del califato (WINTER y MARGOLIN, 2017: 26). Además, el grupo ensalza el valor de las musulmanas que ejercieron misiones suicidas en los casos de Irak y Chechenia.

El grupo se muestra tan abierto a que esta posibilidad surja que, incluso, creó al menos una escuela, al-Zawra, para proporcionar, entre otras labores, formación militar a las mujeres que integraban el califato (DAVIS, 2018). El Ejército Libre Sirio (ELS) también denunció la creación de un batallón compuesto por 200 mujeres cuyo objetivo era llevar a cabo misiones suicidas en los territorios controlados por el grupo. Existen pocos datos sobre mujeres que hayan llevado a cabo operaciones de martirio en nombre de Daesh pero al menos una mujer consiguió inmolarse en una base de las fuerzas kurdas en Kobane en el año 2014 (BBC monitoring, 2014). Este hecho fue posteriormente reivindicado por el grupo en la serie Stories of the Mujahideen (JAWAD AL-TAMIMI, 2017). Otra mujer tunecina fue detenida en el año 2014 portando un cinturón explosivo en Bab al-Hawa, cruce entre Siria y Turquía (BBC monitoring, 2014).

De ser ciertos, estos ataques no suponen una novedad en un grupo yihadista y están en consonancia con la línea iniciada por al-Zarqawi y con la que Daesh comulga. Resulta factible pensar que las actuaciones llevadas a cabo por estas mujeres respondían también a una necesidad del grupo debido a la zona donde se libraba la batalla y en la que Daesh contaba con menos recursos. Más innovadores son los datos recogidos por fuentes secundarias y que apuntan a que durante los primeros años de vida del califato las mujeres han podido frecuentar el campo de batalla. Se tiene constancia de que al menos una mujer de origen canadiense enviaba tuits desde zonas donde Daesh ha librado cruentos combates como Kobane o Deir Ezzor. En estos mensajes deja claro que "los insurgentes la trataban con el respeto que merece una hermana" (TRAC, 2014). Su presencia no obstante, podía estar relacionada con otro tipo de actividades como es el suministro de víveres. La detención de la viuda de un alto mando de Daesh conocida como Umm Sayyaf continuó reforzando esta hipótesis de que las mujeres estaban más involucradas en la militancia armada. Sayyaf aseguró que Daesh cuenta con todo un comando de mujeres en labores de inteligencia (YOUSSEF y HARRIS, 2015). 
Tras el recrudecimiento de la guerra a finales de 2016 el grupo cambia su concepto de estrategia ofensiva a defensiva y emplaza por primera vez y de manera abierta a las mujeres a la lucha. El llamamiento a las armas tiene su precedente en diciembre de 2016 en el noticiero semanal al-Naba, también del grupo y cuyo idioma de difusión es el árabe. En el recuerda que pese a que la yihad no es una obligación para las mujeres, las musulmanas deben saber que si el enemigo entra, la yihad es tan necesaria para ellas como para los hombres y debe repeler el ataque con cualquiera de los medios disponibles (WINTER y MARGOLIN, 2017: 24). Este mensaje se traslada a su público occidental en el número 11 de Rumiyah en el que la organización anima a las mujeres al final del ensayo Our journey to Allah a "levantarse con coraje y sacrifico en esta guerra, no por la falta de hombres sino por su amor a la yihad". El artículo, que sale a la luz en julio de 2017, utiliza a referentes clásicos como Umm Umara para justificar su participación en la lucha armada. Conforme iba avanzando la contienda, el grupo llegó a recrudecer aún más su mensaje señalando que "era necesario y obligatorio para las mujeres luchar en la yihad" (DEARDEN, 2017). Un hecho que supone el quebrantamiento de una de sus normas sociales básicas.

En junio de 2017 varios medios de comunicación occidentales publicaron que 38 mujeres se habían inmolado en Mosul. Un total de 20 lo hicieron entre el 2 de julio de 2017 y el 5 del mismo mes como último esfuerzo para tratar de frenar las tropas iraquíes (ENSOR, 2017). Estas operaciones, no obstante, no han sido reivindicadas por el grupo terrorista (WINTER y MARGOLIN, 2017: 23) y según un informe del Instituto Nacional de Estudios de Seguridad Israelí (INSS) tan solo 7 han podido ser verificadas (SCHWEITZER y MENDELBOIM, 2018). Una de estas misiones pudo registrarse gracias a una instantánea tomada momentos antes de que una mujer se inmolara acompañada de un niño pequeño. Esta hizo estallar una bomba que llevaba debajo del niqab justo cuando pasaba delante de los miembros de seguridad iraquíes (BLOOM y COTTEE, 2017). El acto supone una evolución con respecto a $\mathrm{AQI}$ ya que solamente Boko Haram ha llegado a utilizar a mujeres con sus hijos en una ofensiva. ELS denunció también el uso de mujeres suicidas en la provincia de Deir EzZor en 2019 (ABU AL-KHAIR, 2019).

Diversas fuentes apuntan a que una mujer de origen ruso combatió como francotiradora en la batalla de Mosul (OLTERMANN y HAWRAMY, 2017) y varias mujeres de diferentes nacionalidades fueron encontradas en un bunker con un gran arsenal de explosivos (SLAWSON, 2017). Asimismo, algunos corresponsales señalaron también que había mujeres luchando de manera activa en el campo de batalla de la ciudad Siria de Deir Ezzor 
(MCKERNAN, 2017). Estos datos supusieron un revulsivo para la comunidad académica, que se mostró escéptica por el origen de las fuentes y pidió cautela (LAHOUD, 2017). Sin embargo, el grupo confirmó este rumor con la difusión en febrero de 2018 de un vídeo a través de su aparatado de propaganda en el que podía verse a mujeres luchando junto a hombres en un campo de batalla (DEARDEN, 2018).

Se trata de la primera vez en la historia que un grupo yihadista emitía imágenes de mujeres participando activamente en una contienda y empuñando armas. El vídeo llegó a través de Al Hayat Media Center y mostraba a mujeres usando niqab, por lo que a diferencia de otros vídeos protagonizados por hombres no podía extraerse la identidad de estas. El documento hacía alusión a al-Zarqawi y señalaba que las mujeres estaban luchando para vengar la pérdida de honor sufrido por "sus hermanas musulmanas" debido a los abusos de la comunidad internacional y del régimen de Bashar al-Asad. Además, reconocía con esta participación "la entrada en una nueva era" (PEARSON, 2018). Las evidencias que respaldan esta nueva tendencia han quedado registradas en más de un documento visual. En marzo de 2019 la agencia Amaq News difundió un vídeo en el que se ve a mujeres luchando en al Baguz, uno de los últimos bastiones de Daesh (ENSOR y LUHN, 2019). Un insurgente ruso también inmortalizó esta lucha grabando a una mujer en primera línea de combate. La militante se dirige a la cámara señalando que "las balas de estos infieles son débiles". El hombre que capta la escena ensalza el valor de la mujer con la siguiente afirmación: "estas hermanas son más útiles que algunos hermanos". ${ }^{4}$

Esta incursión supone la entrada en una nueva era para la violencia política ejercida por una mujer en un grupo yihadista tal y como remarca el mismo Daesh en su aparato de propaganda. Se trata de un deseo cumplido por parte de las mujeres seguidoras de esta corriente yihadista $y$, más concretamente, de los postulados de Daesh. Estas han mostrado su predisposición y deseo de poder luchar y utilizar armas asegurando que su único freno residía en contar con el beneplácito de la organización. Así lo han manifestado en sus perfiles sociales varias jóvenes occidentales que llevaron a cabo la migración (HOYLE et. al., 2015). El grupo se ha hecho eco de estas aspiraciones a través del semanal Al-Naba (LAHOUD, 2017) y lo han registrado periodistas después de entrevistarse con mujeres desplazadas a los territorios controlados por el grupo (THOMPSON, 2016).

4 Perfil de Twitter @alg_hismendi, (2019, 21 de marzo), el vídeo puede verse en la red social Twitter el siguiente enlace: https://twitter.com/werej_1/status/1 108738701406949377 (última consulta: 24 de diciembre de 2019). 


\section{LAS FUERZAS KURDAS DE SIRIA}

Los kurdos están considerados como el mayor grupo étnico sin estado del mundo y su población está repartida entre Turquía, Irán, Irak o Siria, país en el que alcanza el 10\% de la población (The World Factbook, 2019). El movimiento nacional kurdo existe en el país desde hace al menos un siglo e históricamente ha estado más fragmentado que sus homólogos en las sociedades turcas e iraquíes (SZEKELY, 2019: 7). Las divisiones entre zonas urbanas y rurales durante el periodo otomano y francés en Siria impidieron el surgimiento de un movimiento político unificado. No es hasta el año 2003 cuando se forma el Partido de la Unión Democrática Siria (PYD, en sus siglas en kurdo), con claros vínculos al Partido de los Trabajadores del Kurdistán (PKK, en sus siglas en turco). Su brazo armado en la guerra se constituyó como las Unidades de Protección Popular (YPG) y las Unidades de Protección Femenina (YPJ, en sus siglas en kurdo).

EI PYD aboga por un modelo político regido por la igualdad de género. El feminismo se ha convertido en la bandera identificativa de la constrainsurgencia y son numerosos los medios de comunicación occidentales que se han hecho eco de esta inclusión en las filas del grupo. Sin embargo, este componente identitario en torno a la ideología de género está presente en el movimiento desde el comienzo gracias a sus raíces marxista-leninistas (DAVIS, 2017). El líder teórico del movimiento, Abdullah Öcalan, considera que la opresión y la injusticia social están relacionadas directamente con la jerarquía de género y apuesta por la liberación de las mujeres como medio para poder superarlas (SIMSEK y JONGERDEN, 2018: 13). De hecho, Öcalan propone una percepción nueva a través de 'jinoloji', un concepto que se construye a partir de la palabra jin (mujer en kurdo) y loji (del griego razón) y que se traduciría como la ciencia de la mujer.

Estas han jugado un rol importante en la política kurda a lo largo de la historia. Öcalan funda junto a su mujer, Kesire Yildirim, el PKK y cuenta desde sus orígenes con mujeres entre sus miembros. La presencia femenina está garantizada en diferentes roles dentro del campo de la logística, de la propaganda o la financiación e incluso en posiciones de liderazgo estratégico (DAVIS, 2017: 79). En los órganos mixtos del PKK y el PYD existe además una cuota de género y los puestos de liderazgo deben estar compuestos, al menos, por un $40 \%$ de mujeres (DE JONG, 2016). No obstante, hay autores que señalan que la sociedad kurda ha estado tradicionalmente dominada por una estructura patriarcal (TASTEKIN, 2014) en la que es bastante común para las mujeres, por ejemplo, contraer matrimonio a corta edad. Luchar se ha convertido para muchas de ellas en la manera de escapar de los roles tradicionales que se presupone para ellas (LAZARUS, 2019). 
El brazo armado kurdo se creó en el año 2012 con el objetivo prioritario de defender a su población de los ataques de Daesh y del régimen de Bashar al-Asad. En esos primeros meses se establecieron comandos mixtos para todos los que querían alistarse. Es tras la incorporación de mujeres combatientes y experimentadas del PKK en el año 2013 cuando finalmente se crean las Unidades de Protección Femenina (YPJ) (SZEKELY, 2019: 8). Según algunos autores, con la intención de romper con la jerarquía de género imperante en los grupos mixtos (DE JONG, 2016) y de evitar el acoso sexual que sufrieron las primeras milicias mixtas del PKK (CRAGIN y DALY, 2009: 67). La brigada no solamente está formada por kurdas sino que también cuenta con una presencia minoritaria de europeas, estadounidenses o árabes de las regiones liberadas de Daesh (LAWRENCE, 2019). Es difícil estimar el número aproximado de combatientes ya que no existen datos debidamente contrastados, aunque algunas fuentes han apuntado que son 10.000 las mujeres que sirven a la causa kurda (COGHLAN, 2016).

Durante el conflicto las mujeres han tenido un rol operativo también importante y hay varios ejemplos de ello. La contienda de Kobane fue liderada por la comandante Meysa Abdo, conocida por el nombre de guerra de $\mathrm{Na}$ arin Afrin (GORMAN, 2017: 32). No es la única figura femenina relevante del conflicto, la comandante Rojda Felat también lideró la campaña para expulsar a Daesh de Raqa. Felat tenía a su mando un contingente mixto de 15.000 combatientes kurdos y árabes. Además, también contaba con el respaldado de las fuerzas especiales de Estados Unidos (COGHLAN, 2016).

Las operaciones de suicidio han sido una de las tácticas empleadas de forma habitual durante las ofensivas. Así, fuentes kurdas señalan que de los primeros 20 ataques que llevó a cabo el grupo durante la batalla de Kobane, 10 eran mujeres. Las mismas fuentes confirman que durante el año 2013 se realizaron 700 atentados suicidas en nombre de las YPG, 200 de los cuales fueron protagonizados por mujeres. La primera en inmolarse fue la joven Selmo Guliselmo en el año 2012 en la ciudad de Alepo (TASTEKIN, 2014). Sin embargo, esta táctica no es una novedad para los kurdos, el Partido de los Trabajadores del Kurdistán creó en el año 1996 un ala militar compuesta por mujeres para llevar a cabo principalmente ataques suicidas.

La mayoría de estos primeros ataques fueron protagonizados de manera desproporcionada por mujeres, un 73\% del total (CRAGIN y DALY, 2009: 67). A este respecto, la investigadora Whaley Eager manifestó que estas provenían, principalmente, de familias con escasos recursos y solían ser reclutadas con 10 años. A esa edad comenzaban un entrenamiento alejadas de su familia con el fin último de dar la vida por la causa kurda (EAGER, 
2008). Estos abusos también han sido puestos en evidencia en el conflicto sirio. Así, en el año 2014 The Syrian Network for Human Rights, reveló el secuestro y el reclutamiento forzado de 69 mujeres, 34 menores de edad, por las milicias kurdas (SN4HR, 2015).

Las mujeres han demostrado a lo largo de la historia ser más efectivas perpetrando ataques suicidas que los hombres debido a que su uso ha sido inesperado por los soldados o los cuerpos de seguridad. Gracias a este factor sorpresa las mujeres cuentan con un mayor índice de letalidad que sus compañeros varones (O'ROURKE, 2009: 682). Además, su participación en actos violentos también adquiere mayor cobertura en los medios de comunicación, circunstancia que es aprovechada por los grupos para lograr notoriedad (SPECKHARD, 2008: 42). El rol de mujeres combatientes dentro de las fuerzas kurdas ha sido el principal mensaje propagandístico difundido por la contrainsurgencia a los medios de comunicación occidentales. La caracterización que se ha hecho de ellas ha sido de valientes e independientes, al contrario que la imagen que se ha trasladado de las mujeres que se han unido a Daesh. La cobertura que se ha hecho de estas últimas ha potenciado su imagen de víctimas, difundiendo reportajes que en gran medida versaban sobre su papel como "las novias de la yihad" (SJOBERG, 2018: 303).

No ha sido el único mensaje que han transmitido. Existen también vídeos, documentales y reportajes exponiendo que los miembros del grupo terrorista Daesh tienen la creencia de que no podrán acceder al Paraíso si les mata una mujer. ${ }^{5}$ Se han recogido varios testimonios apuntando que estos huyen despavoridos si son conscientes de que se trata de una mujer la que empuña un arma. "Monitoreamos sus llamadas de radio. Cuando escuchan la voz de una mujer se ponen histéricos" (TASTEKIN, 2014). El destacamento de mujeres kurdas iraníes también es consciente de esta ventaja operativa e incluso han llegado a entonar canciones por los altavoces antes de entrar en batalla con el objetivo de desestabilizarlos (DEHGHANPISHEH y GEORGY, 2016).

\section{Otras brigadas femeninas en la guerra siria}

La presencia femenina en el campo de batalla no se ha limitado en el conflicto sirio a Daesh y a las fuerzas kurdas sino que hay al menos otros dos actores relevantes que cuentan con mujeres en sus filas. Estos han experimentado menor trascendencia mediática en Occidente pero también son

5 Uno de estos documentales puede verse en https://www.youtube.com/watch? $v=y b c s R b h n p n c$ 
relevantes a la hora de analizar el papel que está teniendo la mujer en la contienda y cuál es el propósito de esta repentina apuesta por la inclusión. A continuación, se analizan las brigadas femeninas del todavía presidente de la República, así como las del Ejército Libre Sirio.

\section{Las leonas de al Asad para la Defensa Nacional}

Las mujeres sirias han estado marginadas de la esfera política y del gobierno del país. La primera vez que una mujer fue electa para el Parlamento fue en el año 1973. En la actualidad, las mujeres ocupan 31 de los 250 escaños de dicha institución y solo el $5 \%$ ha alcanzado un puesto en algún ministerio (CHARLES y DENMAN, 2012: 202). No reflejan datos mejores la representación femenina dentro del poder judicial. Hasta 1975 las mujeres tenían prohibido ejercer la abogacía y en el año 2010 solamente el $13 \%$ de los jueces y los fiscales del país eran mujeres (UNICEF, 2011). Asimismo, la discriminación legislativa contra las mujeres en el país aún sigue evidente en derechos relacionados con la libre circulación por el territorio nacional, el reparto equitativo de la herencia o la poligamia (ALSABA y KAPILASHRAMI, 2016: 9). Las Fuerzas Armadas sirias cuentan con cientos de mujeres en el Ejército pero la mayoría ocupan puestos administrativos que no están muy bien remunerados (ATASSI, 2012).

Tras el estallido de la guerra Bashar al-Asad tampoco ha demostrado respeto por los derechos de las mujeres sirias. Así, diversos organismos se han encargado de denunciar las agresiones sexuales que han padecido cientos de mujeres en el conflicto por parte del régimen, que incluso emplea la violación como medio de tortura en las cárceles, en redadas dentro sus propios hogares o en puestos de control (HRW, 2012). Un informe del Consejo de Derechos Humanos de las Naciones Unidas del año 2018 recoge decenas de testimonios de mujeres que han sufrido este tipo de violencia. Una mujer denuncia en este mismo documento que un grupo de soldados de las fuerzas gubernamentales entraron en su casa en Homs y violaron a su hija. Tras matarla a ella y a su marido, la madre también acabó siendo violada por dos soldados (HRC, 2018: 7).

Dentro de este contexto, la Guardia Republicana Siria crea en el año 2013 el primer batallón femenino conocido como Las Leonas para la Defensa Nacional (SZERLY, 2019). Se trata de un cuerpo de élite cuya principal función es proteger los puestos de control fronterizos. En el año 2013 el 5\% de los soldados que ocupaban los puestos de control eran mujeres (SOHLMAN, 2013) y estas en raras ocasiones han sido vistas en primera línea de fuego (VOA News, 2013, 5 de junio). La formación está compuesta por unos 800 soldados pertenecientes, en su mayoría, a la minoría alauí. No es la 
única facción, en el año 2017 el gobierno crea un nuevo batallón llamado Jansawat Suriya. Está formado por unas 150 mujeres de la ciudad de Qamishli, en el norte del país (MCKERNAN, 2017).

Algunos autores señalan que la repentina incorporación responde más a una estrategia del presidente de la República que a una verdadera política de inclusión, sobre todo debido al contexto expuesto en Siria (SZEKELY, 2019). Al-Asad, con este viraje, está utilizando a las mujeres para promover una imagen de modernidad con el objetivo de diferenciarse de uno de sus máximos oponentes en el campo: Daesh (LEDUC, 2015). Asimismo, en la esfera mediática también necesita hacerle frente a la contrainsurgencia kurda. Para lograrlo varios medios afines al régimen han publicitado la labor de estas mujeres y en Youtube se pueden encontrar una gran variedad de vídeos que muestran entrevistas y grabaciones de estas entrenando, montando camiones y utilizando armas, generalmente con música (SCOTT, 2014).

Asimismo, otra de las razones que apuntan a esta nueva inclusión de las mujeres dentro del Ejército sirio se debería a la escasez de hombres dentro del cuerpo. Entre las deserciones, que comenzaron al poco de iniciarse las hostilidades en el año 2011 y las bajas producidas durante la guerra, que ascendían a unas 44.000 en el año 2014, el presidente ha visto mermado el número de sus fuerzas de 325.000 a 150.000 (KOZAK, 2014). Con estos datos el régimen estableció una campaña de reclutamiento desesperada, deteniendo incluso a jóvenes universitarios en Damasco o Dera y promulgando varios decretos para restringir la capacidad de los hombres en edad militar de abandonar el país (KOZAK, 2014).

\section{Ejército Libre Sirio: Nuestra madre Aicha}

Un grupo de oficiales desertores del Ejército sirio creaba el 29 de julio de 2011 el Ejército Libre Sirio (ELS). El coronel Riad al-Asad se situaba a la cabeza de la formación y declaraba que la misión de la fuerza armada era combatir al régimen de Bashar al-Asad y proteger a la ciudadanía de las masacres de las que estaba siendo objeto. Asimismo, el coronel también hacía un llamamiento animando a miembros del Ejército a que desertaran y se unieran a su causa (LAYLA, 2013). Zubaida al-Meeki fue la primera mujer con rango de oficial que desertaba de las filas de las fuerzas gubernamentales y se insertaba en el ELS en el año 2012. Al-Meeki, perteneciente a la minoría alauí, declaró públicamente que le era imposible permanecer en el Ejército sirio después de los crímenes que estaba perpetrando el régimen (ATASSI, 2012). Se unió a la oposición en un pueblo al sur de Damasco después de que el ELS tomara el control de la mayor parte de esta región (ATASSI, 2012). 
Al-Meeki nunca ha participado en un combate y su principal función en las filas del ELS consiste en entrenar a los nuevos combatientes voluntarios que se unían sin ningún tipo de bagaje castrense. La oficial preparó, por ejemplo, a los miembros de la brigada Yund Ala (Soldados de Alá) para entrar en batalla pese a que en Siria no sea frecuente que las mujeres entrenen a los hombres. Tan solo un año más tarde de su deserción comenzaron a aparecer varias brigadas femeninas vinculadas al ELS. Una de las más importantes es Nuestra Madre Aicha, que debe su nombre a la segunda mujer del profeta Mahoma y que lideró la conocida como batalla del Camello (656).

La brigada está constituida, principalmente, por mujeres universitarias del país y es una unidad que funciona de manera independiente en el aspecto formal. Entre sus funciones destaca proporcionar ayuda a la población civil, también están a cargo de dos hospitales de campaña para los heridos y tienen presencia en una estación de policía para mujeres detenidas (Reuters, 2015). En una sociedad patriarcal, donde los hombres y las mujeres suelen contar con espacios separados para evitar interacciones, resulta indispensable contar con presencia femenina para conseguir llegar a toda la población sin quebrantar las costumbres culturales. No es la única brigada femenina en la oposición, aunque el resto cuenta con un número menor de militantes y son más explícitas con su ideario islamista (HEFFEZ, 2013). Por ejemplo, la brigada femenina Sumayya bint Jayyat, ${ }^{6}$ aliada con la ELS, publicó en su perfil de Facebook una imagen de una mujer armada con banderas sirias en la que podía leerse su oposición a "los enemigos del Islam", catalogados como judíos, comunistas, rusos e infieles. ${ }^{7}$

También se dieron a conocer durante el conflicto la brigada suní Ikhlas, operativa en Alepo. Pese a que existen vídeos en los que se las puede ver ejerciendo labores de combate, lo cierto es que su función principal es llevar a cabo registros a otras mujeres en puestos fronterizos. La brigada Daughters of al-Walid en Homs, anunció en el momento de su fundación a través de YouTube que su misión era "cuidar a los heridos y ayudar a los refugiados". Miembros de las dos brigadas coinciden en que la brutalidad

6 La carga simbólica del nombre elegido para la brigada da buena cuenta de su base doctrinal ya que se trata de la primera mujer que se convirtió en mártir en tiempos del Profeta.

7 Perfil de Facebook @ktybtSmyhBntAlkhyat, (3013, 10 de marzo), el enlace para la página puede encontrarse aquí: https://www.facebook.com/KtybtSmyhBntAlkhyat/photos /a.594228527273470/631933690169620/?type=3\&theater (última consulta 24 de diciembre de 2019). 
del régimen y las agresiones sexuales que han sufrido las mujeres les han obligado a desafiar las normas tradicionales, apoyar la lucha armada así como aprender a defenderse (HEFFEZ, 2013).

Sin embargo, un ex oficial del Ejército sirio, que se unió a los rebeldes, señala en una entrevista que es escéptico sobre el valor que las mujeres tienen en los puestos de primera línea de combate y considera que su papel es, en gran medida, simbólico. El hombre pregunta de manera retórica al periodista que si se imaginaba a las mujeres peleando (SOHLMAN, 2013). Un activista de Homs también señalaba que el papel de las mujeres es puramente ornamental. "Los vídeos de brigadas femeninas a veces tienen la intención de avergonzar a los hombres para que se movilicen y participen en la lucha" (ATASSI, 2012). Este es el caso de un vídeo producido por facciones del ELS en el que puede verse a una mujer con un arma pronunciando un discurso en el que afea la conducta de los hombres que no están participando en las hostilidades. ${ }^{8}$

\section{CONCLUSIONES}

Los cuatro principales grupos que han sido objeto de análisis tienen diferentes enfoques en cuanto a la política de género, pero lo cierto es que su apuesta por la incursión femenina parece responder más a razones estratégicas o prácticas que a una verdadera búsqueda de igualdad entre hombres y mujeres. Así, pese a que Daesh se ha convertido en la primera organización yihadista que permite a sus mujeres entrar en batalla, esta circunstancia solamente se ha producido cuando el fin del califato estaba cerca y necesitaban recursos para poder defenderse.

Las mujeres kurdas se han convertido en un símbolo feminista en la esfera occidental gracias a la cobertura mediática que ha tenido su rol como combatientes y a la propaganda vertida por la contrainsurgencia kurda. Esta exposición mediática potenciando el lado femenino de las hostilidades no tiene precedentes en la organización y ha logrado construirle al grupo una narrativa para diferenciarse de Daesh, uno de sus grandes enemigos en el combate así como contar con una estrategia para desestabilizar su amenaza en el campo de batalla.

Por su parte, el gobierno de Bashar al-Asad no podía quedarse al margen de lo que parece una competición por trasladar a la audiencia una imagen

8 Sham qamshlo Sham, (2012, 20 de octubre), el vídeo se encuentra en https://www.youtube. com/watch?v=z1U2D4Ufdq8 (última consulta 24 de diciembre de 2019). 
de inclusión en la esfera internacional. Las políticas del régimen en cuanto a las mujeres, la obvia discriminación institucional y las agresiones que han sufrido durante la contienda son un claro indicador de que las razones para incluirlas en su Ejército responden más a una campaña de marketing que a una nueva política de igualdad. De hecho, si el presidente de la República considerara a estas capacitadas para el combate hubiera extrapolado los decretos promulgados para el servicio militar también a las mujeres.

Asimismo, hay otra razón que explica este florecimiento de las brigadas femeninas. Tal y como ha quedado patente a lo largo del artículo, la sociedad siria se rige por unas costumbres claramente conservadoras, aún más potenciadas en el caso de los grupos islamistas. Estos necesitan emplear a las mujeres para asegurarse que las normas patriarcales por las que se rigen no corren el riesgo de quebrantarse. Por eso, tal y como se ha descrito, el principal papel de las mujeres es abastecer a la población o hacer registros en los puestos de control. Este papel que asumen las mujeres en las filas de la oposición no tiene por qué estar reñido con su deseo de participar en la contienda en la medida que sus convicciones se lo permitan.

Por último, es pertinente señalar que queda mucho por conocer acerca de las motivaciones que han llevado a estas mujeres a participar en cada uno de los grupos que se han analizado, así como el papel que han tenido dentro de los mismos. La posguerra traerá consigo las verdaderas consecuencias de esta inclusión y se podrá observar si su rol estuvo motivado por las circunstancias y las dinámicas de la guerra o si era el comienzo de una ruptura con las costumbres que imperan en la sociedad y puedan liderar la construcción de una Siria en paz.

\section{Bibliografía}

ABINGTON, Timothy (2019), "Armies of women: The Syria crisis and the new war thesis", E-international Relations Students [https://www.e-ir. info/2019/03/27/armies-of-women-the-syria-crisis-and-the-new-warthesis/]

AL-MUHAJIRAH, Umm Summayyah (2015), "A jihad without fighting", From the battle of Al-Ahzab to the war of coalitions, Dabiq, al Hayat Media Center, $n^{\circ} 11$, pp.40-45.

AL-KHAIR, Waleed (2019, 2 de febrero), "Isis deploys female bombers in last Syria bastion", Diyaruna [https://diyaruna.com/en_GB/articles/ cnmi_di/features/2019/02/15/feature-02].

AL- AZMI, Hamad; ZAKARIYA, Ahmad (2014, 29 de octubre), "Syria's FSA officer says Kuwaiti women fighting alongside Islamic State", Al Watan (recuperado con BBC Monitoring). 
ALSABA, Khuloud; KAPILASHRAMI, Anuj (2016), "Understanding women's experience of violence and the political economy of gender in conflict: the case of Syria", Reproductive Health Matters, vol. 24:47, pp.5-17

ÁlVAREZ-OSSORIO, Ignacio (2016), Siria, revolución, sectarismo y yihad, Los Libros de la Catarata, Madrid.

ATASSI, Basma (2012, 23 de octubre), "Defected women general trains Sirya's rebels", Al Jazeera [https://www.aljazeera.com/indepth/features/2012/10/20121022105057794364.html]

AVILÉS, Juan, (2017), Historia del terrorismo yihadista: de Al Qaeda a Daesh, Síntesis, Madrid.

BLOOM, Mia (2011), Bombshell: Women and terrorism, University of Pennsylvania Press, Filadelfia.

BLOOM, Mia y COTTEE, Simon (2017, 8 de septiembre), "The myth of the ISIS Female Suicide Bomber", The Atlantic [https://www.theatlantic.com/ international/archive/2017/09/isis-female-suicide-bomber/539172/].

CHARLES, Lorraine y DENMAN, Kate (2012), "Every knot has someone to undo it. Using the capabilities approach as a lens to view the status of women leading up to the Arab Spring in Syria", Journal of International Women's Studies, vol. 13:5.

CHINKIN, Christine y KALDOR, Mary (2013), "Gender and new wars", Journal of International Affairs, vol. 67:1, pp.167-187

COGHLAN, Tom (2016), "Revolutionary kurdish feminist leads assault on Raqqa", The Times [https://www.thetimes.co.uk/article/revolutionary-kurdish-feminist-leads-assault-on-raqqa-29kdsrgpc].

CRAGIN, Kim y DALY, Sara (2009), Women as terrorists, Mothers, recruiters, and martyrs, Preager, California.

CUNNINGHAM, Karla J. (2003), "Cross-Regional Trends in Female Terrorism", Studies in Conflict \& Terrorism, vol, 26:3, pp.171-195.

DAVIS, Jessica (2017), Women in modern terrorism: from liberation wars to global jihad and the Islamic State, Rowman \& Littlefiel, Maryland.

DAVIS, Jessica (2018), "Women and the Isil phenomenon: from recruiters to suicide bombers and everything between" [https://www.researchgate.net/ publication/322505279_Women_and_the_ISIL_phenomenon_from_recruiters_to_suicide_bombers_and_everything_in_between].

DE CATALDO, Luisella y TIZIANA, Valentini (1992), Il filo di Arianna. Donne, eversione armata e pentitismo, CEDAM, Padua.

DE JONG, Alex (2016), "¿A commune in Rojava?", New Politics, vol. XV:4 [https://newpol.org/issue_post/commune-rojava/]. 
DEARDEN, Lizzie (2017, 6 de octubre), "Isis calls on women to figth and launch terror attacks for the first time", Independent [https://www.independent. co.uk/news/world/middle-east/isis-war-syria-iraq-women-call-to-arms-islamic-state-terror-attacks-propaganda-change-ban-frontline-a7986986.html].

DEARDEN, Lizzie (2018, 8 de febrero), "Isis propaganda video shows women fighting for the first time amid desperation to bolster ranks", Independent [https://www.independent.co.uk/news/world/middle-east/isis-video-women-jihadis-female-fighters-recruitment-syria-iraq-islamic-state-propaganda-a820062 1.html].

DEHGHANPISHEH, Babak y GEORGY, Michael (2016, 3 de noviembre), "Kurdish women fighters battle Islamic State with machineguns and songs", Reuters [https://www.reuters.com/article/us-mideast-crisis-mosul-womenfighters/kurdish-women-fighters-battle-islamic-state-with-machineguns-and-songs-idUSKBN12Y2DC].

DJAMILA, Danièle y ABU-HAIDAR, Farida (1999), "Women and politics in Argelia from the war of independence to our day", Research in African Literatures, vol. 30:3, pp. 62-77, Indiana University Press.

DOOLEY, Stacey (2018, 6 de marzo), "Meet the former sex slaves fighting IS on the front line", BBC [https://www.bbc.co.uk/bbcthree/article/647a4bda-f52b-4642-a39b-bde3e6ec8f14].

EAGER, Paige (2008), From freedom fighters to terrorists: women and political violence, Routledge, Londres.

ENSOR, Josie (2017, 10 de julio), "Chilling picture shows female Isil fighter holding child moments before detonating suicide vest", The Telegraph [https://www.telegraph.co.uk/news/2017/07/08/chilling-picture-shows-female-isil-fighter-holding-child-moments/].

ENSOR, Josie y LUHN, Alec (2019, 22 de marzo), "Women take to the front lines as desperate Isil fights to the end", The Telegraph [https://www. telegraph.co.uk/news/2019/03/22/isil-relaxes-rules-let-women-takearms-jihadists-final-stand/].

FREEDOM HOUSE (2010, marzo), "Women's Rights in the Middle East and North Africa 2010 - Syria" [https://www.refworld.org/docid/4b9901 ldc.html].

GHAZZAWI, Razan (2014, 8 de abril), "Seeing the women in revolutionary Syria", Open Democracy [https://www.opendemocracy.net/en/north-africa-west-asia/seeing-women-in-revolutionary-syria/]

GORMAN, Shawn. E (2017), Are female counterinsurgency units effective? A case study of the female kurdish militias of Iraq and Syria, Georgetown University, Tesis de Licenciatura. 
HAUSMANN, Ricardo; TYSON Laura D. y ZAHIDI, Saadia (2011), "The Global Gender Gap Report", World Economic Forum [http://reports.weforum.org/global-gender-gap-2011/].

HEFFEZ, Adam (2013, 29 de julio), "When women joined the jihad in Syria", Haaretz [https://www.haaretz.com/opinion/.premium-adam-heffez-women-warriors-in-syria-1.53 14117 ].

HOYLE, Carolyn; BRADFORD, Alexandra y FRENETT, Ross (2015) "Becoming Mulan? Female Western Migrants to ISIS", Institute for strategic Dialogue [https://www.isdglobal.org/wpcontent/uploads/2016/02/ ISDJ2969_Becoming_Mulan_01.15_WEB.pdf ].

HUFF, Zach, (2017, julio), "Exclusive: Christian Women Up Against ISIS", Clarion Project [https://clarionproject.org/christian-women-against-isis/].

HUMAN RIGHTS WATCH (2012, junio), Syria: sexual assault in detention [https://www.hrw.org/news/2012/06/15/syria-sexual-assault-detention].

HUMAN RIGHTS WATCH [HRW], World report 2019: events of 2018 [https://www.hrw.org/sites/default/files/world_report_download/hrw_ world_report_2019.pdf].

HUMAN RIGHTS COUNCIL (HRC) (2018, marzo), "I lost my dignity": sexualy and gender-based violence in the Syrian Arab Republic, A/HRC/37/ CRP.3

JAWAD AL-TAMIMI, Aymenn (2016, 17 de octubre), "The Archivist: Stories of the Mujahideen: Women of the Islamic State", Jihadology [http://www. aymennjawad.org/19291/the-archivist-stories-of-the-mujahideen-women].

JEANNE, Billie (2017), "Revolutionary damascene roses: women and media in the Syrian conflict", en PROFANTER, Annemarie y MAESTRI, Elena (eds.), Arab women and the media in changing landscapes: realities and changes, Palgrave Macmillan, Londres, pp. 229-244.

KOZAK, Christopher (2014, diciembre), "The Assad Regime Under Stress: Conscription and Protest among Alawite and Minority Populations in Syria", Institute for the Study of War [http://iswresearch.blogspot.com/2014/12/ the-assad-regime-under-stress.html].

LAHOUD, Nelly (2017), "Can women be soldiers of the Islamic state?", Survival. Global Politics and Strategy, vol. 59:1, pp. 61-78.

LAYLA, Karel, (2013), "Mothers at home and activists on the street?". The role of women in the Syrian revolution of 2011-2012, MIR, vol 2:3, pp. 16. LAWRANCE, Joey $(2019,26$ de junio), "Faces of war: Kurdistan's armed struggle against Islamic State", The Guardian [https://www.theguardian.com/world/2019/jun/26/faces-of-war-kurdistan-armed-struggle-against-islamic-state]. 
LAZARUS, Sarah (2019, 27 de enero), "Women. Life. Freedom. Female fighters of Kurdistan", CNN [https://edition.cnn.com/2019/01/27/homepage2/kurdish-female-fighters/index.html].

LUCAS, Scott (2014, 9 de abril), "Syria video: women, join the militia!", EA World Video [https://eaworldview.com/2014/04/syria-video-women-join-militia/].

MCDONALD, Eileen (1991), Shoot the Women First, Random House, Nueva York.

MCKERNAN, Bethan (2017, 2 de febrero), "Syrian army creates new women's unit to fight Isis", Independent [https://www.independent.co.uk/ news/world/middle-east/syrian-army-creates-womens-unit-fighting-isis-islamic-state-bashar-al-assad-regime-terror-group-a7558896.html] .

NASSER, Rabie y MEHCHY, Zaki (2012), "Determinants of Labour Force Participation in Syria (2001-2010)", Economic Research Forum, Working Paper $n^{\circ} 698$ [http://erf.org.eg/publications/determinants-labor-force-participation-syria-2001-2010/].

O'ROURKE, Lindsey (2009), "What's Especial about Female Suicide Terrorism?", Security Studies, vol. 18:4, pp.681-718

OLTERMANN, Philip y HAWRAMY, Fazel (2017, 19 de julio), "Suspected Isis fighter seized in Mosul may be missing German girl, 16", The Guardia[https://www.theguardian.com/world/2017/jul/19/isis-mosul-missing-german-girl-linda-wenzel].

REUTERS, (2015, 4 de enero), "Politics civil unrest conflict" [https://pictures.reuters.com/archive/SYRIA-CRISIS-GM1EB 1507BX01.html].

RUMIYAH (2017, julio) "Our journey to Allah", The ruling on ghanimah, fay and ihtitab, Rumiyah, al Hayat Media Center, n¹1, p.12-15

SCHWEITZER, Yoram y MENDELBOIM, Aviad (2018), "Report on suicide attacks in 2017: Fewer attacks, more women bombers", Instituto of National Securities Studies (INSS), n 1008 [http://www.inss.org.il/publication/ report-suicide-attacks-2017-fewer-attacks-women/].

SIMSEK, Bahar y JONGERDEN, Joost (2018), "Gender revolution in Rojava: The voices beyond tabloid geopolitics", Geopolitics.

SJOBER, Laura. (2018), "Jihadi brides and female vounteers: reading the Islamic State's war to see gender and agency in conflict dynamics", Conflict and management and peace science, vol. 35:3, pp. 296-311.

SLAWSON, Nicola (2017, 22 de julio), "German girls arrested in Mosul is missing Linda Wenzel, say authorities", The Guardian [https://www. theguardian.com/world/2017/jul/22/german-girl-arrested-mosul-isis-missing-linda-wenzel-16]. 
SOHLMAN, Eva (2013, 11 de abril), "Sisters in arms join the fighting in Syria", The New York Times [https://www.nytimes.com/2013/04/11/ world/middleeast/sisters-in-arms-join-the-fighting-in-syria.html].

SPECKHARD, Anne (2008), "The emergence of female suicide terrorists", Studies in Conflict and Terrorism, vol. 31:11, pp. 995-1023.

SYRIAN NETWORK FOR HUMAN RIGHTS (SN4HR) (2015), Syrian women in the eye of the storm [http://sn4hr.org/wp-content/pdf/english/Syrian_women_in_the_center_of_the_hurricane_en.pdf].

SZELKY, Ora (2019), "Fighting about women: ideologies of gender in the Syrian civil war", Journal of Global Security Studies, Oxford University Press, pp. 1-19

TASTEKIN, Fehim (2014, 8 de octubre), "Kobani is becoming Turkey's war", Al Monitor [https://www.al-monitor.com/pulse/originals/2014/10/ turkey-syria-kurds-isis-kobani-war.html].

TERRORISM RESEARCH \& ANALYSIS CONSORTIUM (2014), From Canada to the Islamic State: a Canadian woman on the frontlines with Isis [https://www.trackingterrorism.org/article/canada-islamic-state-canadian-woman-frontlines-isis ].

THOMSON, David, (2016), Les Revenants, Le Seuil, París.

UNICEF (2016), "A profile of child marriage in the Middle East and North Africa" [https://www.unicef.org/mena/media/2641/file/MENA-ChildMarriageReport.pdf.pdf].

UNICEF (2011), "SYRIA, MENA Gender Equality Profile. Status of girls and Women in the Middle East and North Africa" [https://www.unicef.org/ gender/files/Syria-Gender-Eqaulity-Profile-2011.pdf].

VICTOR, Barbara (2004), Army of Roses. Inside the World of Palestinian Women Suicide Bombers, Constable \& Robinson, Londres.

VINOGRADOVA, Lyuba (2017), Ángeles vengadores: las francotiradoras soviéticas en la Segunda Guerra Mundial, Pasado \& Presente. Barcelona. VOA NEWS (2013, 5 de junio), "Women a Small, Symbolic Part of Syrian Fighting Force" [https://www.voanews.com/world-news/middle-east-dontuse/women-small-symbolic-part-syrian-fighting-force].

WINTER, Charlie y MARGOLIN, Devorah (2017, 3 de agosto), "The mujahidat dilemma: Female combatants and the Islamic State", CTC Sentinel, pp. 23-33.

YEGINSU, Ceylan (2018, 26 de julio), "Mary Ellis, who flew british spitfires in World War II, diez at 101", The New York Times [https://www. nytimes.com/2018/07/26/obituaries/uk-mary-ellis-dead.html]. 
YOUSSEF, Nancy y HARRIS, Shanae (2015, 7 de mayo), "The women who secretly keep Isis running", The Daily Best [https://www.thedailybeast. com/the-women-who-secretly-keep-isis-running].

ZAMAN, Amberi, (2017, 6 de noviembre), "Syria's Arab, Kurdish women join forces to fight for future", Al Monitor [https://www.al-monitor.com/ pulse/originals/2017/11/syria-raqqa-fight-kurds-women-sdf-islamic-state. html\#ixzz5t67cZRDO]. 